\title{
Optical vortices in twisted elliptical optical fibers with torsional stress
}

\author{
Constantine Alexeyev, ${ }^{1}$ Elena Barshak, ${ }^{1}$ Yurii Fridman, ${ }^{1}$ and Maxim Yavorsky ${ }^{1,2, *}$ \\ ${ }^{1}$ Taurida National V.I. Vernadsky University, Vernadsky Prospekt, 4, Simferopol, 95007, Crimea, Ukraine \\ ${ }^{2}$ Univ. Bordeaux, LOMA, UMR 5798, F-33400 Talence, France; CNRS, LOMA, UMR 5798, F-33400 Talence, France \\ ${ }^{*}$ Corresponding author: maxyavorsky@yahoo.com
}

\begin{abstract}
In the present paper we have derived the analytical expressions for the modes of twisted elliptical fibers with torsional mechanical stress at various relationships of the fiber parameters. It was shown that circularly polarized optical vortices with the topological charges \pm 1 can propagate in elliptical fibers as generic modes if ellipticity and the twist induced circular birefringence suppress the spin orbit inter action. A comparison of the obtained results with the corresponding results for spun elliptical fibers is made.
\end{abstract}

OCIS codes: $\quad 060.2400,060.2310,260.6042$.

\section{Introduction}

Optical fibers with the torsional mechanical stress induced by twisting a fiber around its axis hold a special place among other types of optical fibers due to their unique property of significant reduction of the polarization mode dispersion [1-4], which is one of the main causes of pulse lengthening in fiber systems and decreasing of sensitivity of the various transducers [ $\underline{5}-7]$. To date, such fibers have been comprehensively studied in a number of papers. In particular, it was theoretically and experimentally demonstrated that the fundamental modes are the circularly polarized fields propagating with different phase velocities, that is, the twist induces circular birefringence in an optical fiber [5]. This leads to an easily experimentally detectable effect of the rotation of the linearly polarized light launched into the twisted fiber. In the case of the presence of some kinds of optical perturbation (such as anisotropy of the material or anisotropy of the shape of the cross section), the corresponding fundamental modes are found to be elliptically polarized in the local frame tracing the direction of the anisotropy axis []. Efforts have been made to investigate the structure of the higher-order modes of twisted fibers, as well. It was shown [9] that the modes with $l=1$ ( $l$ being the azimuthal mode number) of ideal twisted fibers are represented by two circularly polarized optical vortices (OVs) the fields with the helical structure of their wavefronts-with unity topological charge (the sign of which coincides with the one of polarization) and the conventional $\mathrm{TE}_{0, n}$ and $\mathrm{TM}_{0, n}$ modes ( $n$ being the radial mode number). The corresponding propagation constants were also obtained. Moreover, the coupling of the modes with different azimuthal numbers induced by twisting has been studied [10] and it was demonstrated that the coupled modes obey a kind of selection rule-they must have the same total angular momentum in the direction of propagation. Quite recently, the effect of small anisotropy on the modes of twisted fibers near the points of instability (the points, in which the degeneracy of the propagation constants takes place) was considered [11]. It was theoretically shown that such fibers can be used for highly effective and stable generation of radially and azimuthally polarized vector beams.

Meanwhile, as far as we know, the effect of anisotropy, in particular the elliptic shape of the fiber's 
cross section, on the structure of the higher-order modes of twisted fiber at arbitrary fiber parameters has not yet been studied. At the same time, apart from the obvious importance of considering such a problem from the fundamental point of view, a purely practical interest in this question is connected with the problem of OV propagation in optical fibers. OVs have a number of unique properties that make them the very promising tools for encoding and transmitting information [12-13], for trapping and manipulating microparticles [14], for astronomy purposes [15-16], etc. To transmit OVs over distance, various types of optical fiber have been proposed [17-21]. However, the mutual influence of torsional mechanical stress (twisting) and anisotropy on the propagation of OVs in optical fibers have not been considered. Thus, in this paper, we pursue two main goals. The first one is to establish and analyze the higher-order $l=1$ modes of twisted elliptical fibers allowing for the vectorial nature of light. The second one is to study whether OVs can propagate in such fibers without changing their form, i.e., as the generic modes.

\section{Model and Perturbation Theory Approach}

The refractive index of the model under consideration is

$$
\begin{aligned}
\hat{n}^{2}(r, \phi)= & n^{2}(r) \hat{1}-2 n_{\mathrm{co}}^{2} \Delta \delta r f_{r}^{\prime} \cos 2 \phi \hat{1} \\
& +q p_{44} n_{\mathrm{co}}^{4} r\left(\begin{array}{ccc}
0 & 0 & \sin \phi \\
0 & 0 & -\cos \phi \\
\sin \phi & -\cos \phi & 0
\end{array}\right)
\end{aligned}
$$

Here the first term describes the axially symmetric distribution of the refractive index in the ideal fibers, $n^{2}(r)=n_{\mathrm{co}}^{2}(1-2 \Delta \cdot f(r)), \Delta=\left(n_{\mathrm{co}}^{2}-n_{\mathrm{cl}}^{2}\right) / 2 n_{\mathrm{co}}^{2}$ is the height of the refractive index profile, $n_{\mathrm{co}}$ and $n_{\mathrm{cl}}$ are the values of the refractive indices in the core and cladding, respectively, and, for a fiber with step-like distribution of the refractive index, the profile function reads as $f(r)=\theta\left(r / r_{0}-1\right), \theta$ being the unity step. The second term in Eq. (1) is connected with the ellipticity of the fiber's cross section [22]. Indeed, the most simple way of introducing the ellipticity is to make the scale transformation: $x \rightarrow x(1+\delta), \quad y \rightarrow y(1-\delta)$, where the ellipticity parameter $\delta \ll 1$, and then expand the refractive index in $\delta$. The prime stands for derivative with respect to $r$. The last term in Eq. (1) appears due to the mechanical stress through the photoelastic effect and $p_{44}=0.5\left(p_{11}-p_{12}\right)$, where $p_{11}$ and $p_{12}$ are the photoelastic constants, and $q=2 \pi / H$ ( $H$ being the pitch of twist). Cylindrical polar coordinates $(r, \phi, z)$ are implied and the axis $z$ is the fiber's axis. Note that the tensor in Eq. (1) is represented in the Cartesian basis: $\mathbf{E}=\operatorname{col}\left(E_{x}, \bar{E}_{y}, E_{z}\right)$, where $\mathbf{E}$ is the electric field.

To get the modes of twisted elliptic fibers, let us consider the vector wave equation for nonmagnetic anisotropic media in the case of the refractive index in Eq. (1) [18]:

$$
\begin{gathered}
\left(\nabla^{2}+k^{2} \hat{n}^{2}(r, \phi)\right) \mathbf{E}(r, \phi, z)=-\nabla\left(\left(\mathbf{E} \cdot \nabla \ln n^{2}(r)\right)\right. \\
\left.+q p_{44} n_{\mathrm{co}}^{4} r\left(\sin \phi \frac{\partial E_{x}}{\partial z}-\cos \phi \frac{\partial E_{y}}{\partial z}\right)\right)
\end{gathered}
$$

where $\nabla=(\partial / \partial x, \partial / \partial y, \partial / \partial z), k=2 \pi / \lambda$, and $\lambda$ is the wavelength in vacuum. The second term in the right-hand side of Eq. (2) emerges due to the tensorial nature of Eq. (1) and it was taken into account that the refractive index can be presented in the form $\hat{n}^{2}(r, \phi)=\varepsilon \hat{1}+\delta \hat{\varepsilon}$. In addition, we have disregarded the influence of ellipticity in the gradient term. It is worth noting that the gradient term $\nabla(\mathbf{E}$. $\left.\nabla \ln n^{2}(r)\right)$ describes the spin-orbit interaction (SOI) of light in an ideal fiber.

Due to the translation invariance of Eq. (1) in the $z$ direction, it is possible to seek a solution in the factorized form: $\mathbf{E}=\mathbf{e}(r, \phi) e^{i \beta z}$, where $\beta$ is the propagation constant. Substituting this into Eq. (2), we can bring this equation to the form of the eigenvalue equation:

$$
\left(\hat{H}_{0}+\hat{V}_{e l l}+\hat{V}_{\mathrm{tw}}\right)|\psi\rangle=\beta^{2}|\psi\rangle,
$$

where the ket-vector $|\psi\rangle=\operatorname{col}\left(e_{x}, e_{y}, e_{z}\right)$, the operator $\hat{H}_{0}$ is responsible for the formation of the modes of ideal optical fibers with accounting the gradient term [22], $\hat{V}_{\text {ell }}=-2 k^{2} n_{\mathrm{co}}^{2} \Delta \delta r f_{r}^{\prime} \cos 2 \phi \hat{1}$ describes the effect of ellipticity on the light propagation, and

$$
\begin{aligned}
& \hat{V}_{\mathrm{tw}}=k^{2} q p_{44} n_{\mathrm{co}}^{4} r\left(\begin{array}{ccc}
0 & 0 & \sin \phi \\
0 & 0 & -\cos \phi \\
\sin \phi & -\cos \phi & 0
\end{array}\right)+ \\
& \quad+i q p_{44} n_{\mathrm{co}}^{2} \beta\left(\begin{array}{ccc}
0.5 \sin 2 \phi r \nabla_{r}-\sin ^{2} \phi \nabla_{\phi} & -1-\cos ^{2} \phi r \nabla_{r}+0.5 \sin 2 \phi \nabla_{\phi} & 0 \\
1+\sin ^{2} \phi r \nabla_{r}+0.5 \sin 2 \phi \nabla_{\phi} & -0.5 \sin 2 \phi r \nabla_{r}-\cos ^{2} \phi \nabla_{\phi} & 0 \\
i r \beta \sin \phi & -i r \beta \cos \phi & 0
\end{array}\right)
\end{aligned}
$$

is aroused due to twisting. 
Taking into consideration that the operators in Eq. (3) have the following orders: $\hat{H}_{0} \propto k^{2} n_{\text {co }}^{2}$, $\hat{V}_{\text {ell }} \propto k^{2} n_{\mathrm{co}}^{2} \Delta \delta, \hat{V}_{\mathrm{tw}} \propto k^{2} q p_{44} n_{\mathrm{co}}^{4} r_{0}\left(r_{0}\right.$ being the core's radius) and that typically $n_{\mathrm{co}}=1.5, \Delta=10^{1}-10^{3}$ (for weakly guiding fibers), $\delta=10^{1}-10^{2}, p_{44}=$ -0.075 (for silica), and $H>10^{3} \mathrm{~m}$, it is easy to see that, for solving Eq. (3) one can use the perturbation theory approach, treating $\hat{H}_{0}$ as a zero-order operator and the operator $\hat{V}=\hat{V}_{\text {ell }}+\hat{V}_{\text {tw }}$ as a perturbation. As is well known [23], the key point of this method is that the system could be described with the so-called perturbation matrix, which has to be built by averaging the operator $\hat{H}=\hat{H}_{0}+\hat{V}$ over the eigenstates $\left|\psi_{l, n}^{(0)}\right\rangle_{i}$ of the zero-order operator $\hat{H}_{0}\left|\psi_{l, n}^{(0)}\right\rangle_{i}=\beta_{i}^{(0)^{2}}\left|\psi_{l, n}^{(0)}\right\rangle_{i}$. Since we are interested in modes with $l=1$, it is somewhat convenient to choose as the zero-order modes the following fields: where the radial function for the step-index fibers is

$$
F_{l, n}(r)=\left\{\begin{array}{l}
\frac{J_{l}\left(\tilde{U}_{n} R\right)}{J_{l}\left(U_{n}\right)}, R \leq 1, \\
\frac{K_{l}\left(\tilde{W}_{n} R\right)}{K_{l}\left(\tilde{W}_{n}\right)}, R \geq 1,
\end{array}\right.
$$

and $R=\frac{r}{r_{0}}$ [24]. The modes $\left|\psi_{1, n}^{(0)}\right\rangle_{1} \equiv|1,1, n\rangle$ and $\left|\psi_{1, n}^{(0)}\right\rangle_{2} \equiv|-1,-1, n\rangle$ are right and left circularly polarized OVs, where the first index describes the sign of polarization, while the second one specifies topological charge; the mode $\left|\psi_{1, n}^{(0)}\right\rangle_{3} \equiv\left|T E_{0, n}\right\rangle$ represents the standard transverse electric mode and the mode $\left|\psi_{1, n}^{(0)}\right\rangle_{4} \equiv\left|\mathrm{TM}_{0, n}\right\rangle$ is the transverse magnetic mode.

The perturbation matrix is:

$$
H_{i j}={ }_{i}\left\langle\psi_{l, n}^{(0)}|\hat{H}| \psi_{l, n}^{(0)}\right\rangle_{j},
$$

where the standard definition of the scalar product is implied:

$$
\langle\Phi \mid \Psi\rangle=\int_{0}^{\infty} \int_{0}^{2 \pi}\left(\begin{array}{lll}
\Phi_{x}^{*} & \Phi_{y}^{*} & \Phi_{z}^{*}
\end{array}\right)\left(\begin{array}{l}
\Psi_{x} \\
\Psi_{y} \\
\Psi_{z}
\end{array}\right) r \mathrm{~d} r \mathrm{~d} \phi .
$$

Using Eqs. (3)-(므), we obtain the perturbation operator for $l=1$ modes:

$$
\hat{H}=\left(\begin{array}{cccc}
\tilde{\beta}_{n}^{2}+A_{n}+C_{n} & 0 & i D_{n} & -D_{n} \\
0 & \tilde{\beta}_{n}^{2}+A_{n}-C_{n} & -i D_{n} & -D_{n} \\
-i D_{n} & i D_{n} & \widetilde{\beta}_{n}^{2} & 0 \\
-D_{n} & -D_{n} & 0 & \tilde{\beta}_{n}^{2}+2 B_{n}
\end{array}\right)
$$

$$
\begin{aligned}
& \left|\psi_{1, n}^{(0)}\right\rangle_{1}=\left(\begin{array}{c}
F_{1, n}(r) \\
i F_{1, n}(r) \\
\frac{i}{\hat{\beta}_{1, n} r}\left[r F_{1, n}^{\prime}-F_{1, n}\right] e^{i \phi}
\end{array}\right) e^{i \phi}, \\
& \left|\psi_{1, n}^{(0)}\right\rangle_{2}=\left(\begin{array}{c}
F_{1, n}(r) \\
-i F_{1, n}(r) \\
\frac{i}{\hat{\beta}_{1, n} r}\left[r F_{1, n}^{\prime}-F_{1, n}\right] e^{i \phi}
\end{array}\right) e^{i \phi}, \\
& \left|\psi_{1, n}^{(0)}\right\rangle_{3}=\left(\begin{array}{c}
-F_{1, n}(r) \sin \phi \\
F_{1, n}(r) \cos \phi \\
0 \\
0 \\
F_{1, n}(r) \cos \phi \\
F_{1, n}(r) \sin \phi \\
\frac{i}{\beta_{1, n} r}\left[r F_{1, n}^{\prime}+F_{1, n}\right]
\end{array}\right),
\end{aligned}
$$

where the SOI constants for the step-index fibers are $A_{n}=\frac{\Delta}{Q_{n} r_{0}^{2}}\left(F_{n} F_{n}^{\prime}-F_{n}^{2}\right)_{R=1}, \quad B_{n}=\frac{\Delta}{Q_{n} r_{0}^{2}}\left(F_{n}^{2}+F_{n} F_{n}^{\prime}\right)_{R=1}$, $Q_{n}=\int_{0}^{\infty} R F_{n}^{2}(R) \mathrm{d} R, \tilde{\beta}_{l, n}$ is the well-known scalar propagation constant [22], $C_{n}=-2\left(\Sigma_{n}+\Theta \beta-\frac{\Theta \beta^{2}}{\beta_{n}}\right)$ is proportional to the twist, $\Sigma_{n}=\frac{k^{2} q\left|p_{44}\right| n_{\mathrm{co}}^{4}}{\beta_{n}}$, and $\Theta=q\left|p_{44}\right| n_{\mathrm{co}}^{2}, D_{n}=-k^{2} n_{\mathrm{co}}^{2} \Delta \delta / Q_{n}$ is the ellipticity constant. Here and in what follows the azimuthal number is omitted and assumed to be unity.

The structure of modes of the fiber and the corresponding propagation constants can be obtained by solving the eigenvalue equation:

$$
\hat{H} \mathbf{x}=0 .
$$

Here $\hat{H}=\hat{H}-\beta^{2}$ and the vectors $\mathbf{x}_{k}$ have the components $\alpha_{i}^{k}$, which are the coefficients of the decomposition of the desired modes over the zero-order fiber modes: $|\psi\rangle_{k}=\sum_{i} a_{i}^{k}\left|\psi_{n}^{(0)}\right\rangle_{i}$. Strictly speaking, solving Eq. (7) with the matrix in Eq. (6) would give the modes of twisted elliptic fibers at arbitrary reasonable 
values of ellipticity, the twist pitch, and the SOI. However, it seems that such a solution would be cumbersome for further analysis. To avoid such a problem, it seems reasonable to consider some particular cases that could be of practical relevance.

\section{Higher-Order $l=1$ Modes: the Case of a Strong Ellipticity}

Let us first consider the case where the constant of ellipticity is much greater than the constants of the SOI and the twist-induced circular birefringence: $\left|D_{n}\right| \gg\left|A_{n}\right|,\left|B_{n}\right|,\left|C_{n}\right|$. Applying the perturbation approach to Eq. (7) and treating the operator $\hat{H}\left(A_{n}=\right.$ $B_{n}=C_{n}=0$ ) as a zero-order one and the operator $\hat{V}=\operatorname{diag}\left(A_{n}+C_{n}, A_{n}-C_{n}, 0,2 B_{n}\right)$ as a perturbation, we arrive at the following expressions for the $l=1$ modes of strongly elliptical fibers with torsional stress:

$$
\begin{aligned}
& |\Psi\rangle_{1}=F_{1, n}(r) \cos \phi\left(\begin{array}{c}
\sin \gamma-\cos \gamma \\
i(\sin \gamma+\cos \gamma)
\end{array}\right), \\
& |\Psi\rangle_{2}=F_{1, n}(r) \cos \phi\left(\begin{array}{c}
\cos \gamma+\sin \gamma \\
i(\cos \gamma-\sin \gamma)
\end{array}\right), \\
& |\Psi\rangle_{3}=F_{1, n}(r) \sin \phi\left(\begin{array}{c}
\sin \gamma-\cos \gamma \\
i(\sin \gamma+\cos \gamma)
\end{array}\right), \\
& |\Psi\rangle_{4}=F_{1, n}(r) \sin \phi\left(\begin{array}{c}
\cos \gamma+\sin \gamma \\
i(\cos \gamma-\sin \gamma)
\end{array}\right),
\end{aligned}
$$

where $\tan 2 \gamma=\frac{\left|B_{n}\right|}{\sum_{n}}, 0 \leq \gamma \leq \frac{\pi}{4}$, and we have omitted the small longitudinal component.

Let us briefly analyze the modes obtained in Eq. (8). It is easy to see that, in the general case, the modes are elliptically polarized and the state of polarization is characterized by the parameter $\gamma$. In turn, $\gamma$ is determined by the ratio of the constants of the SOI and the twist. Consequently, there are two limiting cases. The first one takes place at $\left|B_{n}\right| \gg\left|\sum_{n}\right|, \sin \gamma, \cos \gamma \rightarrow 1 / \sqrt{ } 2$, and the fields become almost linearly polarized and describe the socalled LP modes [24]. It is known that LP modes are the eigenfields of straight strongly elliptical fibers, so that we have got a correct limit for the mode in Eq. (8) that can be considered as an indirect confirmation of the obtained result. The second limiting case occurs when the twist-induced circular birefringence much exceeds the SOI: $\left|\sum_{n}\right| \gg\left|B_{n}\right|, \sin \gamma \rightarrow 0$, $\cos \gamma \rightarrow 1$, and the modes are almost circularly polarized. This seems to be quite reasonable, if we keep in mind that ellipticity by itself cannot affect the state of polarization. As for the field's dependence on the polar angle through sine and cosine factors, it is worth mentioning that its specific form is determined exactly by the influence of large ellipticity. As a consequence of such an angular dependence, the modes in Eq. (8) do not posses the orbital angular momentum $(\mathrm{OA} M)$ but bear only the spin angular momentum (SAM), which can be easily calculated by making use of the well-known formula [25]: $S_{z} \propto\left\langle\Psi\left|\left(\begin{array}{ll}0 & i \\ i & 0\end{array}\right)\right| \Psi\right\rangle$, where the basis of linear polarization is implied. As an example, the SAM for the mode $|\Psi\rangle_{2}$ reads as $S_{z}=\cos 2 \gamma$.

We failed to provide compact analytical expressions for the propagation constants and only the numerical calculation of the spectrum. This is connected with the fact that the perturbation matrix $\hat{H}$ depends on its eigenvalue $\beta$ through the coefficient $C_{n}$. Figure 1 shows the dependence of the corrections to the scalar propagation constant on the twist. It is seen that the spectral curves corresponding to the modes with the opposite parity of the angular function are strongly spaced by the influence of large ellipticity. Additional repulsion of the propagation constants corresponding to the fields with the orthogonal states of polarization is provided by the mutual influence of the twist-induced circular birefringence and the SOI. Such a behavior of the propagation constants qualitatively coincides with one of the eigenvalues of Eq. (6). This allows one to make a conclusion on the stability of the modes in Eq. (8) with respect to external perturbations. It should be noted that, in real optical fibers, the velocity of the energy and information propagation through the fiber is connected with the group velocity rather than with the phase one, which is determined by propagation constants. In our paper, we consider only the phase velocities of the modes for two reasons. The first is that we are strongly interested in the question of stability of OVs, which is connected with the behavior of the propagation constants. The second reason is that we believe that the problem of influence of twisting and ellipticity of the fiber on the group velocity of OVs is very nontrivial and deserves a separate comprehensive consideration.

\section{Higher-Order $l=1$ Modes: Stable Circularly Polarized Optical Vortices}

Now we will consider the most important case where the effect of the twist and ellipticity of the transverse cross section on light's propagation is much greater

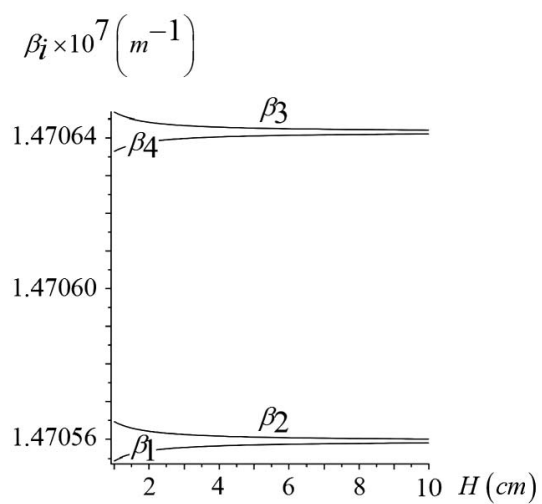

Fig. 1. Dependence of the propagation constants $\beta_{i}$ on the twist $H$ in the case of twisted strongly elliptical optical fibers; the fiber parameters are $n_{\text {co }} 1.48, \Delta \quad 0.001, r_{0} \quad 10 \lambda_{\mathrm{He}-\mathrm{Ne}}, \delta \quad 0.01$, and $p_{44} \quad 0.075$. 
that the one of the SOI: $\left|D_{n}\right|,\left|C_{n}\right| \gg\left|A_{n}\right|,\left|B_{n}\right|$. This means that, following the idea of applying the perturbation method to Eq. (7), we should regard the operator $\hat{H}\left(A_{n}=B_{n}=0\right)$ in Eq. (6) as a zero-order one and the operator $V=\operatorname{diag}\left(A_{n}, \bar{A}_{n}, 0,2 B_{n}\right)$ as a small perturbation. After some algebra, the $l=1$ modes of strongly elliptical intensely twisted fibers are found to be

$$
\begin{aligned}
& |\Phi\rangle_{1}=\frac{1}{\sqrt{ } 2} F_{1}(R)\left(\sin \theta e^{i \phi}-\cos \theta e^{i \phi}\right)\left(\begin{array}{c}
1 \\
i
\end{array}\right), \\
& |\Phi\rangle_{2}=\frac{1}{\sqrt{ } 2} F_{1}(R)\left(\cos \theta e^{i \phi}+\sin \theta e^{i \phi}\right)\left(\begin{array}{c}
1 \\
i
\end{array}\right), \\
& |\Phi\rangle_{3}=\frac{1}{\sqrt{ } 2} F_{1}(R)\left(\sin e^{i \phi}-\cos \theta e^{i \phi}\right)\left(\begin{array}{c}
1 \\
-i
\end{array}\right), \\
& |\Phi\rangle_{4}=\frac{1}{\sqrt{ } 2} F_{1}(R)\left(\cos \theta e^{i \phi}+\sin \theta e^{i \phi}\right)\left(\begin{array}{c}
1 \\
-i
\end{array}\right),
\end{aligned}
$$

where $\tan 2 \theta=\frac{\left|D_{n}\right|}{\Sigma}, 0<\theta<\frac{\pi}{4}$. It may seem strange that there are no constants of the SOI in these expressions. It turns out that the spectrum of the zero-order operator $\hat{H}\left(A_{n}=B_{n}=0\right)$ is not degenerate as long as $\Sigma_{n}, D_{n} \neq 0$. Thus, one can disregard the influence of the perturbation $\hat{V}$ on the mode structure. This is valid under the following two conditions:

$$
\sin \theta \gg \frac{\left|B_{n}\right|}{2\left|D_{n}\right|}, \quad \frac{4 \Sigma_{n}}{\left|B_{n}\right|} \gg 1 .
$$

Let us discuss the fields in Eq. (9). It is seen that they are circularly polarized, which is obviously connected with the influence of large twist-induced birefringence against the background of small SOI. The main feature of the modes in Eq. (9) is that they are represented by a weighted superposition of two OVs with the opposite signs of their topological charges. It is well known that such a superposition presents an OV with a well-defined topological charge, which coincides with the charge of the partial OV with the largest weight coefficient. Naturally, this holds as long as weight coefficients in such a superposition are different. It is easy to understand that the topological charges of the modes in Eq. (9) are $l_{1,3}=-1, l_{2,4}=1$ and these values remain the same as the parameter $\theta$ varies within its valid region. Such a behavior is an example of the topological stability of OVs. Nevertheless, changing the parameter $\theta$ (through fiber parameters) manifests itself in changing such a fundamental characteristic of the field as the OAM. Indeed, it is known that, in the general case, the OAM is not fully determined by the topological charge as it takes place in the simplest cases. Using the well-known expression $L_{z} \propto$ $\left\langle\Psi\left|-i \frac{\partial}{\partial \phi}\right| \Psi\right\rangle[25]$, it is straightforward to show that the modes in Eq. (9) have the following OAM:

$$
L_{z}^{1,3}=-\cos 2 \theta, \quad L_{z}^{2,4}=\cos 2 \theta,
$$

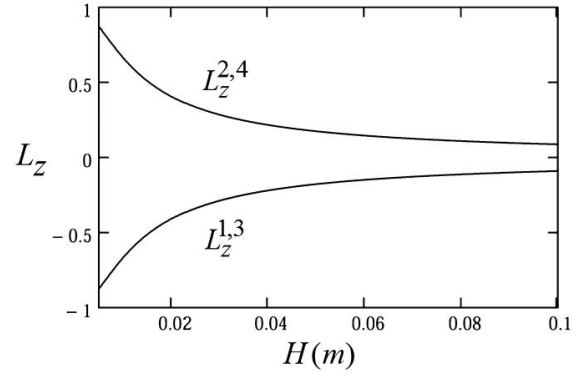

Fig. 2. OAM in the direction of propagation (in relative units) $L_{z}$ of the vortex modes [Eq. (11)] versus the twist pitch $H$; the fiber parameters are $n_{\text {со }} 1.4 \overline{8,} \Delta \quad 0.001, r_{0} \quad 10 \lambda_{\mathrm{He}-\mathrm{Ne}}, \delta \quad 0.01$, $p_{44} \quad 0.075$.

where the upper indices stand for the mode number. Figure 2 presents the dependence of OAM [Eq. (11)] on the twist. It is seen that, as the twist pitch $H \overline{\text { de}}-$ creases, the curves tend to the maximal values \pm 1 . Such a behavior can be easily understood if we consider the corresponding limiting case for the modes in Eq. (9). Indeed, as $\Sigma_{n} \gg\left|D_{n}\right|\left(H \ll \frac{2 \pi k\left|p_{44}\right| n_{\mathrm{co}}^{3}}{\left|D_{n}\right|}\right), \theta \rightarrow 0$, and the modes become almost ideal OVs with the OAM (in relative units) coinciding with their topological charges. Such a regime could be useful when OVs are utilized as information carriers and should have the well-defined OAM. Within this regime, one has to be especially careful with meeting the conditions in Eq. (10) under which the modes in Eq. (9) are valid.

The numerically obtained propagation constants corresponding to modes in Eq. (9) are shown in Fig. 3 . The main point here is that there exists a wide range of the fiber parameters at which all propagation constants (along with the eigenvalues of the operator $\left.\hat{H}\left(A_{n}=B_{n}=0\right)\right)$ are well spaced. Thus, within such a range, the propagation of OVs [Eq. (9)] appears to be robust with respect to small (in comparison with effect of ellipticity and twisting) external perturbations. In other words, circularly polarized OVs are the generic modes of the strongly elliptical intensely

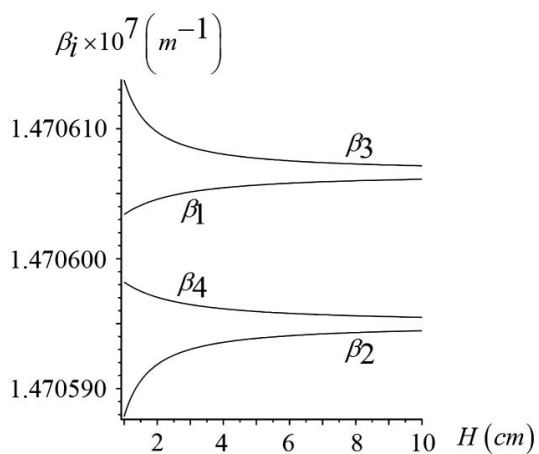

Fig. 3. Dependence of the propagation constants $\beta_{i}$ on the twist $H$ in the case of intensely twisted strongly elliptical optical fibers; the fiber parameters are $n_{c o} 1.48, \Delta \quad 0.001, r_{0} \quad 10 \lambda_{\mathrm{He}-\mathrm{Ne}}$, $\delta \quad 0.01$, and $p_{44} \quad 0.075$. 
twisted optical fibers with torsional mechanical stress. This is the main result of the paper.

\section{Discussion}

In the present paper we have derived the analytical expressions for the modes of twisted elliptical fibers with torsional mechanical stress at different relationships of the fiber parameters. It was shown that OVs are not the modes of twisted elliptical fibers at arbitrary relation of fibers parameters, which is the case for twisted fibers without anisotropy. It was demonstrated that circularly polarized OVs with the topological charges \pm 1 can propagate in elliptical fibers as generic modes when ellipticity and the twistinduced circular birefringence suppress the SOI.

It is interesting to compare the obtained results with the corresponding ones established for spun elliptical fibers [17]. The key difference between these two models is connected with the $z$ dependence of the refractive index in the case of spun fibers provided by the rotation of the ellipse in the fiber cross section around the fiber's axis. This gives rise to the violation of the translation invariance in $z$ coordinate in the wave equation. To overcome this obstacle, as was shown in [17], one has to rewrite the wave equation in a local frame, which traces the rotation of the anisotropy axis coinciding with one of the ellipse axes. Such a procedure allows one to restore the corresponding translation invariance and obtain the modes in the local frame. From a mathematical point of view, to get the expressions for the modes in a global frame, one should apply the $z$-dependent operator of the inversion transformation to the modes in the local frame. Along with shifting the angular variable $\phi \rightarrow \phi-q z$, this gives rise to the effect that each component of the mode acquires its own $z$-dependent phase factor in addition to the global phase of the mode. Physically, it means that the modes of such spun fibers are the analogue of the Bloch wavesthe waves of the following type: $|\psi(r, \phi, z)\rangle=$ $\mathbf{A}(r, \phi, z) e^{i \beta z}$, where $\mathbf{A}(r, \phi, z)=\mathbf{A}(r, \phi, z+H)$ is the periodic vector amplitude. Such a type of the solution obviously reflects the periodicity property of the refractive index of spun fibers: $n^{2}(x, y, z)=n^{2}(x, y, z+H)$.

Meanwhile, from a dynamical point of view, it turns out that the operators describing the rotation of the anisotropy axis in spun fibers and the influence of the mechanical stress in twisted fibers act on $l=1$ modes qualitatively in the same manner - they produce a kind of circular birefringence, leading to the coupling of exactly the same zero-order modes during the construction of the perturbation matrices. This results in the identical structure of the modes of twisted and spun elliptical fibers (at the corresponding fiber parameters), save for the $\phi-q z$ dependence of the angular functions and the Bloch wave nature of the latter.

These distinctions lead to two basic differences in mode properties. The first one is that the intensity distribution of the modes of spun elliptical fibers rotates as light propagates through the fiber. The second one takes place when the vortex-modes regime for twisted and spun fibers is achieved. Indeed, as was demonstrated in [17], at the condition $q \tilde{\beta}_{n} \gg\left|D_{n}\right|$, all four modes of spun fibers, being represented by the circularly polarized OVs, propagate with almost the same phase velocities, “...thus exhibiting one of the main features of propagation in vacuum." As can be easily seen from Fig. $\underline{3}$, this is not the case for twisted elliptical fibers.

Save for the above-mentioned difference in the mode properties, the eigenfields of spun and twisted elliptical fibers at the corresponding fiber parameters have much the same basic characteristics as the state of polarization, topological charge of the OVs, SAM, and OAM.

\section{References}

1. A. J. Barlow, J. J. Ramskov Hansen, and D. N. Payne, "Bire fringence and polarization mode dispersion in spun single mode fibers," Appl. Opt. 20, 29622968 (1981).

2. M. J. Li, X. Chen, and D. A. Nolan, "Effects of residual stress on polarization mode dispersion of fibers made with different types of spinning," Opt. Lett. 29, 448450 (2004).

3. M. Wang, T. Li, and S. Jian, "Analytical theory for polarization mode dispersion of spun and twisted fiber," Opt. Express 11, 24032410 (2003).

4. R. E. Schuh, E. S. R. Sikora, N. G. Walker, A. S. Siddiqui, L. M. Cleeson, and D. H. O. Bebbington, "Theoretical analysis and measurement of effects of fibre twist on polarisation mode dispersion of optical fibres," Electron. Lett. 31, 17721773 (1995).

5. R. Ulrich and A. Simon, "Polarization optics of twisted single mode fibers," Appl. Opt. 18, 22412251 (1979).

6. A. Smith, "Polarization and magnetooptic properties of single mode optical fiber," Appl. Opt. 17, 5256 (1978).

7. T. Yoshino, "Theory for the Faraday effect in optical fiber," J. Opt. Soc. Am. B 22, 18561860 (2005).

8. V. L. Ginzburg, "Investigation of stress by the optical method," Zh. Tekh. Fiz. 14, 181192 (1944) (in Russian).

9. C. N. Alexeyev, A. V. Volyar, and M. A. Yavorsky, "Optical vortices in twisted optical fibres with torsional stress," J. Opt. A 10, 095007 (2008).

10. C. N. Alexeyev, E. V. Borshak, A. V. Volyar, and M. A. Yavorsky, "Angular momentum conservation and coupled vortex modes in twisted optical fibres with torsional stress," J. Opt. A 11, 094011 (2009).

11. C. N. Alexeyev, E. V. Barshak, T. A. Fadeyeva, A. V. Volyar, and M. A. Yavorsky, "Generation of radially and azimuthally polarized beams with elliptical anisotropic twisted optical fibres," J. Opt. 13, 075706 (2011).

12. G. Gibson, J. Courtial, M. Padgett, M. Vasnetsov, V. Pas'ko, S. Barnett, and S. Franke Arnold, "Free space information transfer using light beams carrying orbital angular momen tum," Opt. Express 12, 54485456 (2004).

13. N. J. Cerf, C. Adami, and P. G Kwiat, "Optical simulation of quantum logic," Phys. Rev. A 57, R1477 R1480 (1998).

14. K. T. Gahagan and G. A. Swartzlander, Jr., "Optical vortex trapping of particles," Opt. Lett. 21, 827829 (1996).

15. G. A. Swartzlander, Jr., "The optical vortex coronagraph," J. Opt. A 11, 090422 (2009).

16. G. A. Swartzlander, Jr., E. L. Ford, R. S. Abdul Malik, L. M. Close, M. A. Peters, D. M. Palacios, and D. W. Wilson, "Astronomical demonstration of an optical vortex corona graph," Opt. Express 16, 1020010207 (2008).

17. C. N. Alexeyev and M. A. Yavorsky, "Optical vortices and the higher order modes of twisted strongly elliptical optical fi bres," J. Opt. A. 6, 824832 (2004).

18. C. N Alexeyev, B A. Lapin, and M. A. Yavorsky, "Optical vor tices and topological phase in strongly anisotropic coiled few mode optical fibers," J. Opt. Soc. Am. B 24, 26662675 (2007). 
19. C. N. Alexeyev, B. P. Lapin, and M. A. Yavorsky, "Helical core optical fibers maintaining propagation of a solitary optical vortex, " Phys. Rev. A 78, 013813 (2008).

20. C. N. Alexeyev, T. A. Fadeyeva, B. P. Lapin, and M. A. Yavorsky, "Generation of optical vortices in layered helical waveguides," Phys. Rev. A 83, 063820 (2011).

21. C. N. Alexeyev and M. A. Yavorsky, "Generation and conver sion of optical vortices in long period helical core optical fibers," Phys. Rev. A 78, 043828 (2008).
22. C. N. Alexeyev, A. V. Volyar, and M. A. Yavorsky, "Fiber optical vortices," in Lasers, Optics and Electro Optics Research Trends L. I. Chen, ed. (Nova, 2007), pp. 131223.

23. A. S. Davydov, Quantum Mechanics (Pergamon, 1976).

24. A. W. Snyder and J. D. Love, Optical Waveguide Theory (Chapman and Hall, 1985).

25. M. V. Berry, "Paraxial beams of spinning light," Proc. SPIE 3487, 611 (1998). 